\title{
Two additional advantages of complex $\mu$-bases for non-ruled real quadric surfaces
}

\author{
Ron Goldman ${ }^{\mathrm{a}}$, Xuhui Wang ${ }^{\mathrm{b}}$ \\ ${ }^{a}$ Department of Computer Science, Rice University, Houston, TX 77251, USA \\ ${ }^{b}$ School of Mathematics, Hefei University of Technology, Hefei, Anhui 230009, China
}

\begin{abstract}
Real $\mu$-bases for non-ruled real quadric surfaces have two potential drawbacks. First, the resultant of the three moving planes corresponding to a real $\mu$-basis represents the implicit equation of the quadric surface, but in some cases contains a linear extraneous factor. Second, even when this resultant contains no extraneous factor, this resultant generates only the affine version of the implicit equation. In contrast, the resultant of the two moving planes corresponding to a complex $\mu$-basis always generates the homogenous version of the implicit equation for the quadric surface with no extraneous factors. We illustrate these phenomena here with three examples.
\end{abstract}

Keywords: Quadric surface, complex $\mu$-basis, real $\mu$-basis

Recently Goldman et al. [2] introduced the notion of a complex $\mu$-basis for a real quadric surface. These authors showed how to apply these complex $\mu$-bases to find the implicit equation and inversion formulas for non-ruled real quadric surfaces and mentioned several advantages of their complex $\mu$-bases over real $\mu$-bases for these quadric surfaces. One advantage that they failed to point out is that the implicit equation generated from the resultant of a real $\mu$-basis for quadric surfaces sometimes has an extraneous factor [1], but the implicit equation generated from the resultant of a complex $\mu$-basis always gives the exact implicit equation with no extraneous factors. A second advantage is that even when the real $\mu$-basis generates the implicit equation with no extraneous factors, the real $\mu$-basis generates only the affine version of the implicit equation, whereas the complex $\mu$-basis generates the homogeneous version of the implicit equation. We illustrate these phenomena here with three examples.

Example 1. (The sphere)

Consider the sphere with the affine quadratic parametrization

$$
\mathbf{P}(s, t)=\left(2 s, 2 t, 1-s^{2}-t^{2}, 1+s^{2}+t^{2}\right) .
$$

Email addresses: rng@rice.edu (Ron Goldman), xhw@hfut.edu.cn (Xuhui Wang)

Preprint submitted to Elsevier

August 2, 2015

(C) 2015. This manuscript version is made available under the Elsevier user license http://www.elsevier.com/open-access/userlicense/1.0/ 
A real $\mu$-basis for this parametrization of the sphere is given by the three syzygies corresponding to the moving planes:

$$
x s+y t+(z-w)=0,(z+w) t-y=0,(z+w) s-x=0,
$$

since their outer product

$$
\operatorname{det}\left(\begin{array}{cccc}
\mathbf{i} & \mathbf{j} & \mathbf{k} & \mathbf{l} \\
s & t & 1 & -1 \\
0 & -1 & t & t \\
-1 & 0 & s & s
\end{array}\right)=\mathbf{P}(s, t)
$$

Taking the resultant of these three moving planes gives the expression for the implicit equation of the sphere with a linear extraneous factor:

$$
\operatorname{det}\left(\begin{array}{ccc}
x & y & z-w \\
0 & z+w & -y \\
z+w & 0 & -x
\end{array}\right)=-(z+w)\left(x^{2}+y^{2}+z^{2}-w^{2}\right)
$$

and an extraneous linear factor remains, even after we dehomogenize this expression.

In contrast, to compute a complex $\mu$-basis for the same sphere, we must use a homogeneous parametrization because the base points of the sphere are at infinity. Consider then the homogeneous parametrization:

$$
\mathbf{P}(s, t, u)=\left(2 s u, 2 t u, u^{2}-s^{2}-t^{2}, u^{2}+s^{2}+t^{2}\right) .
$$

The base points of this parametrization are located at $s= \pm \mathrm{i} t, u=0$. A straightforward computation [2] now yields the following two moving planes corresponding to a complex $\mu$-basis for the sphere:

$$
(x+\mathrm{i} y)(s-\mathrm{i} t)+(w-z)(-u),(w+z)(s-\mathrm{i} t)+(x-i y)(-u) .
$$

Taking the resultant of these two moving planes gives the homogenous expression for the implicit equation of the sphere with no extraneous factors:

$$
\operatorname{det}\left(\begin{array}{ll}
x+\mathrm{i} y & w-z \\
w+z & x-\mathrm{i} y
\end{array}\right) \equiv x^{2}+y^{2}+z^{2}-w^{2} .
$$

Notice that even though we have used complex parameters, we do indeed get an implicit equation with real coefficients.

Example 2. (The Hyperboloid of Two Sheets)

Consider the hyperboloid of two sheets with the affine quadratic parametrization

$$
\mathbf{P}(s, t)=\left(1+s^{2}+t^{2}, 2 t, 1-s^{2}-t^{2}, 2 s\right) .
$$

A real $\mu$-basis for this parametrization of this hyperboloid of two sheets is given by the three syzygies corresponding to the moving planes:

$$
w s+y t+(z-x)=0,(x+z) s-w=0,(x+z) t-y=0,
$$


since their outer product

$$
\operatorname{det}\left(\begin{array}{cccc}
\mathbf{i} & \mathbf{j} & \mathbf{k} & \mathbf{l} \\
-1 & t & 1 & s \\
s & 0 & s & -1 \\
t & -1 & t & 0
\end{array}\right)=\mathbf{P}(s, t)
$$

Taking the resultant of these three moving planes gives the expression for the implicit equation of the hyperboloid of two sheets with a linear extraneous factor:

$$
\operatorname{det}\left(\begin{array}{ccc}
w & y & z-x \\
0 & x+z & -y \\
x+z & 0 & -w
\end{array}\right)=-(x+z)\left(y^{2}+z^{2}+w^{2}-x^{2}\right),
$$

and once again an extraneous linear factor remains, even after we dehomogenize this expression.

In contrast, to compute a complex $\mu$-basis for the same hyperboloid of two sheets, we must use a homogeneous parametrization because the base points of the hyperboloid of two sheets are at infinity. Consider then the homogeneous parametrization:

$$
\mathbf{P}(s, t, u)=\left(u^{2}+s^{2}+t^{2}, 2 t u, u^{2}-s^{2}-t^{2}, 2 s u\right) .
$$

The base points of this parametrization are located at $s= \pm \mathrm{i} t, u=0$. A straightforward computation now yields the following two moving planes corresponding to a complex $\mu$-basis for the hyperboloid of two sheets:

$$
(w+\mathrm{i} y)(s-\mathrm{i} t)+(x-z)(-u),(x+z)(s-\mathrm{i} t)+(w-\mathrm{i} y)(-u) .
$$

Taking the resultant of these two moving planes gives the homogenous expression for the implicit equation of the hyperboloid of two sheets with no extraneous factors:

$$
\operatorname{det}\left(\begin{array}{cc}
w+\mathrm{i} y & x-z \\
x+z & w-\mathrm{i} y
\end{array}\right) \equiv y^{2}+z^{2}+w^{2}-x^{2} .
$$

Notice again that even though we have used complex parameters, we do indeed get an implicit equation with real coefficients.

The implicit equation generated from the resultant of the three moving planes corresponding to a real $\mu$-basis for quadric surfaces does not always have an extraneous factor; there are some cases where the real $\mu$-basis gives the exact implicit equation with no extraneous factors. We illustrate such a case here with one additional example.

Example 3. (The Elliptical Paraboloid)

Consider the elliptical paraboloid with the affine quadratic parametrization

$$
\mathbf{P}(s, t)=\left(1+s^{2}+t^{2}, t, s, 1\right) .
$$


A real $\mu$-basis for this parametrization of the elliptical paraboloid is given by the three syzygies corresponding to the moving planes:

$$
t-y=0, s-z=0, z s+y t+1-x=0,
$$

since their outer product

$$
\operatorname{det}\left(\begin{array}{cccc}
\mathbf{i} & \mathbf{j} & \mathbf{k} & \mathbf{l} \\
0 & -1 & 0 & t \\
0 & 0 & -1 & s \\
-1 & t & s & 1
\end{array}\right)=\mathbf{P}(s, t) .
$$

Taking the resultant of these three moving planes gives the expression for the implicit equation of the elliptical paraboloid:

$$
\operatorname{det}\left(\begin{array}{ccc}
0 & 1 & -y \\
1 & 0 & -z \\
z & y & 1-x
\end{array}\right)=x-1-y^{2}-z^{2} .
$$

This expression represents the implicit equation of the elliptical paraboloid in affine space with no extraneous factors.

In contrast, to compute a complex $\mu$-basis for the same elliptical paraboloid, we must use a homogeneous parametrization because the base points are at infinity. Consider then the homogeneous parametrization

$$
\mathbf{P}(s, t, u)=\left(u^{2}+s^{2}+t^{2}, t u, s u, u^{2}\right) .
$$

The base points of this parametrization are located at $s= \pm t \mathrm{i}, u=0$. A straightforward computation now yields the following two moving planes corresponding to a complex $\mu$-basis for the elliptical paraboloid:

$$
(z+\mathrm{i} y)(s-\mathrm{i} t)+(x-w)(-u),(w)(s-\mathrm{i} t)+(z-i y)(-u) .
$$

Taking the resultant of these two moving planes gives the following expression for the homogeneous implicit equation of the elliptical paraboloid:

$$
\operatorname{det}\left(\begin{array}{cc}
z+\mathrm{i} y & x-w \\
w & z-\mathrm{i} y
\end{array}\right) \equiv y^{2}+z^{2}-w x+w^{2} .
$$

Notice yet again that even though we have used complex parameters, we do indeed get an implicit equation with real coefficients. Moreover, the complex $\mu$-basis generates the homogeneous version of the implicit equation, whereas the real $\mu$-basis generates only the affine version of the implicit equation.

\section{Acknowledgements}

This work is partially supported by grants from NSF of China (Nos. 11301131, 11301116, 61272024) and the Fundamental Research Funds for the Central Universities. 


\section{References}

[1] Falai Chen, Liyong Shen, Jiansong Deng, Implicitization and parametrization of quadratic and cubic surfaces by $\mu$-bases. Computing, Vol.79, 131$142,2007$.

[2] Ron Goldman, Thomas W. Sederberg, Xuhui Wang, Complex $\mu$-bases for real quadric surfaces, Computer Aided Geometric Design, Vol.37, 57-68, 2015. 